\title{
Substance Use Disorders in Patients With Parkinson's Disease and Adverse Hospitalization Outcomes: A National Inpatient Study
}

Jaskaranpreet Kaur ${ }^{1}$, Ramneek K. Sandhu ${ }^{2}$, Khadija T. Kubra ${ }^{3}$, Johanna S. Canenguez Benitez ${ }^{4}$, Henry K. Onyeaka $^{5}$, Sabiha Akter ${ }^{6}$, Ozge C. Amuk Williams ${ }^{7}$

1. Internal Medicine, Dayanand Medical College and Hospital, Punjab, IND 2. Internal Medicine, Shri Guru Ram Das Institute of Medical Sciences and Research, Amritsar, IND 3. Internal Medicine, Bangladesh Medical College and Hospital, Dhaka, BGD 4. Internal Medicine, Larkin Community Hospital, Houston, USA 5. Psychiatry, Harvard School of Medicine, Boston, USA 6. Psychiatry, Bergen New Bridge Medical Center, Paramus, USA 7. Psychiatry, Koç University School of Medicine, Istanbul, TUR

Corresponding author: Ozge C. Amuk Williams, drozgeceren@gmail.com

\section{Abstract \\ Objectives}

To understand the demographic pattern of substance use disorders (SUD) in Parkinson's disease (PD) inpatients and to evaluate the impact of SUD on hospitalization outcomes including the severity of illness, length of stay (LOS), total charges, and disposition to nursing facilities.

\section{Methods}

We used the nationwide inpatient sample and identified adult patients (age, $\geqslant 40$ years) with PD as a primary diagnosis and comorbid SUD $(\mathrm{N}=959)$ and grouped by co-diagnosis of alcohol $(\mathrm{N}=789)$, cannabis $(\mathrm{N}=46)$, opioid ( $\mathrm{N}=30)$, stimulants $(\mathrm{N}=54)$ and barbiturate $(\mathrm{N}=40)$ use disorders. We used a binomial logistic regression model to evaluate the odds ratio (OR) for major loss of functioning and disposition to nursing facilities in PD inpatients. All regression models were adjusted for demographics, including age, sex, race, and median household income.

\section{Results}

Alcohol, opioid, and stimulant use disorders were prevalent in old-age adults (60-79 years), males, and whites, but cannabis use was prevalent in middle-aged adults (40-59 years), and barbiturate use among older-age (>80 years). The severity of illness is statistically higher in PD inpatients with comorbid opioid and barbiturate use disorders with major loss of body functioning, closely seconded by alcohol and stimulant use disorder cohorts (27.6\% and $25.9 \%$, respectively). Disease severity and loss of body functioning increase with advancing age (>80 years adults, OR 5.8, 95\%CI 5.32-6.37), and in blacks (OR 1.7, 95\%CI 1.561.81), and those with opioid use disorder (OR 3.8, 95\%CI 1.96-7.35). PD inpatients with barbiturate use disorder had a higher LOS and charges by 17.4 days and $\$ 68,922$, and six-fold increased likelihood (95\%CI 2.33-15.67) for disposition to nursing facilities.

Review began 06/10/2021 Review ended 06/16/2021 Published 06/29/2021

\section{(c) Copyright 2021}

Kaur et al. This is an open access article distributed under the terms of the Creative Commons Attribution License CC-BY 4.0., which permits unrestricted use, distribution, and reproduction in any medium, provided the original author and source are credited.

\section{Conclusions}

SUD is prevalent among PD patients and is associated with more severe illnesses with body loss functioning and prolonged care. A multidisciplinary care model including collaborative neuropsychiatric and addiction management is required to manage SUD among PD patients to lessen disease severity, slow down the disease progression and potentially save medical costs.

Categories: Neurology, Psychiatry, Epidemiology/Public Health

Keywords: parkinson's disease, barbiturate use, opioid use, stimulants use, hospitalization outcomes

\section{Introduction}

Parkinson's disease (PD) is the second most prevalent multisystem synucleinopathic neurodegenerative disorder in North America [1]. The national center for health statistics center estimates the incidence of PD to rise to nearly one million by 2020 and 1.25 million by 2030 in the United States (US) owing to the rapidly growing aging population [2]. PD is characterized by a constellation of motor and non-motor symptoms owing to the degeneracy and depletion of dopaminergic neuronal in the cardio and nephritic vasculature, central (CNS), peripheral (PNS), and enteric nervous systems [3,4].

PD is a disease with no cure or prevention that often begins with minimal prodromal symptoms but ultimately manifests into a myriad of disabling symptomatology progressively increasing in severity with advancing age [1]. The dopamine receptor 3 (d3), localized within the limbic area, is the primary receptor 
involved in the compulsive perseverance associated with reward and reinforcement loops that mediate drugseeking behaviors amongst substance use disorder patients [4]. Furthermore, recent studies implicate the dopamine receptor $4(\mathrm{~d} 4)$ to be involved in the relapse to stimulant abuse, whilst dopamine receptors 1 and 2 (d1 and d2, respectively) predominantly work within a highly coordinated synaptic network encompassing the prefrontal cortex, hippocampus, and the amygdala [3].

Given the chronicity, progressive clinical deterioration, and gross morbidity of the disease, patients were believed to often resort to absolving their non-motor symptoms by turning to illicit substances that often lead to comorbid substance use disorders (SUD). Novel research findings suggest early-onset illicit substance use and chronic use of stimulants may potentiate the early-onset parkinsonism, eventually leading to the disease [5]. In this study, we seek to look at the five most abused classes of substances among PD patients, including barbiturate, stimulants (cocaine and amphetamine), opioids, and cannabis. We used the nationwide inpatient sample (NIS) to firstly understand the demographic pattern of SUD in PD inpatients, and next to evaluate the odds of association between severity of illness and disposition to nursing facilities in PD inpatients and across the spectrum of SUD.

\section{Materials And Methods \\ Data source and sample}

A retrospective study was conducted using the healthcare cost and utilization project's (HCUP) NIS data for the year 2014. The NIS database is considered to determine patterns in demographics and hospitalization outcomes. It is the largest inpatient database which covers 4,411 hospitals from 44 states in the United States. In order to protect the privacy of individual patients, physicians, and hospitals, the state and hospital identifiers were de-identified [6]. So, we were not required the institutional review board's permission to conduct a study on publicly available de-identified data.

We extracted a sample of 62,784 adult patients (age, $\geqslant 40$ years) with a principal diagnosis of PD based on the International Classification of Diseases, ninth revision (ICD-9) diagnosis code of 332. An analysis of the sample data extrapolated from the NIS found that 959 (1.25\%) patients had comorbid diagnoses of SUD, and was grouped by comorbid alcohol use disorder $(\mathrm{N}=789)$, cannabis use disorder $(\mathrm{N}=46)$, opioid use disorder $(\mathrm{N}=30)$, stimulant use disorders $(\mathrm{N}=54)$ and barbiturate use disorder $(\mathrm{N}=40)$.

\section{Variables of interest}

The demographic variables included in this study were age, sex, race, and median household income. In order to measure the differences in hospital outcomes in PD patients by the presence of comorbid SUD, included the following variables: severity of illness, length of stay (LOS), total charges, and disposition to a skilled or intermediate nursing facility (SNF/INF). In the NIS, all patient refined DRGs (APR-DRGs) are assigned using software developed by $3 \mathrm{~m}$ health information systems to classify the severity of illness into minor, moderate, and major loss of body function. LOS is the number of nights the patient remained in the hospital for the principal diagnosis of PD, and total charges during PD hospitalization do not include professional fees and non-covered charges [7].

\section{Statistical analyses}

We used descriptive statistics and linear-by-linear association tests for categorical data and independent sample t-test for the continuous data to measure the differences in demographics and hospital outcomes across the spectrum of SUD. We used the discharge weight variable, which is given in the NIS, to attain a national representation of the sample population. We used a binomial logistic regression model to evaluate the odds ratio (OR) for major loss of functioning and disposition to SNF/INF in PD inpatients with the spectrum of SUD. All regression models were adjusted for demographics including age, sex, race, and median household income. A P value $<0.05$ was used to determine the statistical significance of the test result. All statistical analyses were done using a Statistical Package for the Social Sciences (SPSS) version 26.0 (IBM Corp., Armonk, NY).

\section{Results}

Alcohol use disorder was most prevalent (82.2\%), followed by stimulants (5.6\%), cannabis (4.9\%), barbiturate (4.2\%), and opioid (3.1\%). Alcohol use disorder was seen in a higher proportion of old-age adults (60-79 years, $68.6 \%)$, male (76.7\%), and white (81.1\%), and equally across all four-percentile median household income groups. Similarly, opioid and stimulant use disorders were found to be highest in old-age adults (60-79 years, $66.7 \%$ and $55.6 \%$ respectively), male (66.7\% and $90 \%$ respectively), and white (83.3\% and $72.2 \%$ respectively), but more amongst those with median household income above 50th percentile. Cannabis use disorder was prevalent in middle-aged adults, 40-59 years (67.4\%), male (55.6\%), and white (86.1\%). PD inpatients with cannabis use disorder were highest amongst those having the lowest income of 0 -25th percentile $(50.0 \%)$. On the contrary, barbiturate use disorder was highly evident amongst older-age adults 80 years and above (62.5\%), male (62.5\%), and white (75.0\%). The demographic distribution of PD inpatients by spectrum of SUD is shown in Table 1 . 


\section{Cureus}

\begin{tabular}{|c|c|c|c|c|c|c|}
\hline Variable & Alcohol & Cannabis & Opioid & Stimulants & Barbiturate & $P$ value \\
\hline Number of inpatients & 789 & 46 & 30 & 54 & 40 & - \\
\hline \multicolumn{7}{|c|}{ Age at admission, in years (\%) } \\
\hline $40-59$ & 18.6 & 67.4 & 16.7 & 44.4 & 0 & \multirow{3}{*}{$<0.001$} \\
\hline $60-79$ & 68.6 & 32.6 & 66.7 & 55.6 & 37.5 & \\
\hline above 80 & 12.8 & 0 & 16.7 & 0 & 62.5 & \\
\hline \multicolumn{7}{|l|}{$\operatorname{Sex}(\%)$} \\
\hline Male & 76.7 & 55.6 & 66.7 & 90.7 & 62.5 & \multirow{2}{*}{$<0.001$} \\
\hline Female & 23.3 & 44.4 & 33.3 & 9.3 & 37.5 & \\
\hline \multicolumn{7}{|l|}{ Race (\%) } \\
\hline White & 81.1 & 86.1 & 83.3 & 72.2 & 75.0 & \multirow{4}{*}{$<0.001$} \\
\hline Black & 7.7 & 0 & 16.7 & 9.3 & 0 & \\
\hline Hispanic & 7.9 & 0 & 0 & 9.3 & 12.5 & \\
\hline Other & 3.3 & 13.9 & 0 & 9.3 & 12.5 & \\
\hline \multicolumn{7}{|c|}{ Median household income, in percentile (\%) } \\
\hline $0-25^{\text {th }}$ & 27.4 & 50.0 & 33.3 & 27.3 & 37.5 & \multirow{4}{*}{$<0.001$} \\
\hline $26^{\text {th }}-50^{\text {th }}$ & 28.1 & 25.0 & 0 & 27.3 & 12.5 & \\
\hline $51^{\mathrm{st}}-75^{\text {th }}$ & 23.8 & 25.0 & 16.7 & 36.4 & 12.5 & \\
\hline $76^{\text {th }}-100^{\text {th }}$ & 20.7 & 0 & 50.0 & 9.1 & 37.5 & \\
\hline \multicolumn{7}{|l|}{ Severity of illness (\%) } \\
\hline Minor loss of function & 22.2 & 43.5 & 0 & 9.3 & 12.5 & \multirow{3}{*}{$<0.001$} \\
\hline Moderate loss of function & 50.2 & 56.5 & 50.0 & 64.8 & 37.5 & \\
\hline Major loss of function & 27.6 & 0 & 50.0 & 25.9 & 50.0 & \\
\hline \multicolumn{7}{|l|}{ Disposition } \\
\hline SNF/IN & 53.4 & 11 & 33.3 & 61.1 & 87.5 & $<0.001$ \\
\hline
\end{tabular}

TABLE 1: Characteristics of Parkinson's disease inpatients by substance use disorders.

SNF/INF: skilled or intermediate nursing facility.

The severity of illness is statistically higher in PD inpatients with comorbid opioid and barbiturate use disorders with major loss of body function, closely seconded by alcohol and stimulant use disorder cohorts (27.6\% and $25.9 \%$, respectively). Loss of body functioning in PD inpatients increases with advancing age as elders ( 80 years and above) have six-fold increased odds (95\% CI 5.32-6.37) compared to $40-59$ years. Males have marginally higher odds (OR 1.2, 95\% CI 1.12-1.22) than females, and blacks have higher odds (OR 1.7, $95 \%$ CI 1.56 - 1.81) than whites for major loss of functioning. Among the SUD, opioid use disorder has the highest odds of association with major loss of functioning in PD inpatients by 3.8 -fold (95\% CI 1.96-7.35) followed by barbiturate use disorder (OR 2.3, 95\% CI 1.23-4.41) and stimulant use disorder (OR 1.9, 95\% CI 1.07-3.38) as shown in Table 2. 


\section{Cureus}

\begin{tabular}{|c|c|c|c|c|}
\hline \multirow{2}{*}{ Variable } & \multirow{2}{*}{ Odds ratio } & \multicolumn{2}{|c|}{$95 \%$ confidence interval } & \multirow{2}{*}{ P-value } \\
\hline & & Lower & Upper & \\
\hline \multicolumn{5}{|c|}{ Age at admission, in years } \\
\hline $40-59$ & Reference & & & \\
\hline $60-79$ & 2.68 & 2.46 & 2.93 & $<0.001$ \\
\hline above 80 & 5.82 & 5.32 & 6.37 & $<0.001$ \\
\hline \multicolumn{5}{|l|}{ Sex } \\
\hline Male & 1.16 & 1.12 & 1.22 & $<0.001$ \\
\hline Female & Reference & & & \\
\hline \multicolumn{5}{|l|}{ Race } \\
\hline White & Reference & & & \\
\hline Black & 1.68 & 1.56 & 1.81 & $<0.001$ \\
\hline Hispanic & 1.06 & 0.97 & 1.12 & 0.191 \\
\hline Other & 1.02 & 0.93 & 1.11 & 0.708 \\
\hline \multicolumn{5}{|c|}{ Median household income, in percentile } \\
\hline $0-25^{\text {th }}$ & Reference & & & \\
\hline $26^{\text {th }}-50^{\text {th }}$ & 0.97 & 0.92 & 1.03 & 0.340 \\
\hline $51^{\text {st }}-75^{\text {th }}$ & 0.99 & 0.94 & 1.05 & 0.802 \\
\hline $76^{\text {th }}-100^{\text {th }}$ & 1.03 & 0.97 & 1.09 & 0.372 \\
\hline \multicolumn{5}{|c|}{ Comorbid substance use disorders } \\
\hline None & Reference & & & \\
\hline Alcohol & 1.57 & 1.32 & 1.86 & $<0.001$ \\
\hline Cannabis & $<0.001$ & $<0.001$ & - & 0.997 \\
\hline Opioid & 3.79 & 1.96 & 7.35 & $<0.001$ \\
\hline Stimulants & 1.90 & 1.07 & 3.38 & 0.029 \\
\hline Barbiturate & 2.33 & 1.23 & 4.41 & 0.009 \\
\hline
\end{tabular}

TABLE 2: Odds of association for major loss of functioning in Parkinson's disease inpatients.

During inpatient management for PD, the LOS varies broadly with comorbid SUD. PD inpatients with barbiturate use disorder had the higher mean LOS and total charges of 17.4 days and $\$ 68,922$, respectively, whereas it was lowest in PD inpatients with opioid use disorder (2.8 days and \$23,311). Elders (80 years and above) have eight-fold increased odds (95\% CI 7.39-8.49) compared to $40-59$ years, and blacks have a 1.5 fold higher odds (95\% CI 1.39-1.60) than white for disposition to SNF/INF. PD inpatients with barbiturate use disorder have six-fold increased likelihood (95\% CI 2.33-15.67) of disposition to SNF/INF, followed by PD inpatient with stimulant (OR 2.6, 95\% CI 1.57-4.39) and alcohol (OR 2.3, 95\% CI 1.97-2.68) use disorders as shown in Table 3. 


\section{Cureus}

\begin{tabular}{|c|c|c|c|c|}
\hline \multirow{2}{*}{ Variable } & \multirow{2}{*}{ Odds ratio } & \multicolumn{2}{|c|}{$95 \%$ confidence interval } & \multirow{2}{*}{ P-value } \\
\hline & & Lower & Upper & \\
\hline \multicolumn{5}{|c|}{ Age at admission, in years } \\
\hline $40-59$ & Reference & & & \\
\hline $60-79$ & 2.95 & 2.76 & 3.15 & $<0.001$ \\
\hline Above 80 & 7.92 & 7.39 & 8.49 & $<0.001$ \\
\hline \multicolumn{5}{|l|}{ Sex } \\
\hline Male & 0.89 & 0.87 & 0.93 & $<0.001$ \\
\hline Female & Reference & & & \\
\hline \multicolumn{5}{|l|}{ Race } \\
\hline White & Reference & & & \\
\hline Black & 1.49 & 1.39 & 1.60 & $<0.001$ \\
\hline Hispanic & 0.77 & 0.71 & 0.83 & $<0.001$ \\
\hline Other & 0.78 & 0.72 & 0.84 & $<0.001$ \\
\hline \multicolumn{5}{|c|}{ Median household income, in percentile } \\
\hline $0-25^{\text {th }}$ & Reference & & & \\
\hline $26^{\text {th }}-50^{\text {th }}$ & 0.92 & 0.87 & 0.97 & 0.001 \\
\hline $51^{\text {st }}-75^{\text {th }}$ & 0.92 & 0.88 & 0.97 & 0.001 \\
\hline $76^{\text {th }}-100^{\text {th }}$ & 0.85 & 0.81 & 0.89 & $<0.001$ \\
\hline \multicolumn{5}{|c|}{ Comorbia suidstance use aisorders } \\
\hline None & Reference & & & \\
\hline Alcohol & 2.29 & 1.97 & 2.68 & $<0.001$ \\
\hline Cannabis & 0.88 & 0.46 & 1.69 & 0.702 \\
\hline Opioid & 0.62 & 0.32 & 1.22 & 0.168 \\
\hline Stimulants & 2.62 & 1.57 & 4.39 & $<0.001$ \\
\hline Barbiturate & 6.04 & 2.33 & 15.67 & $<0.001$ \\
\hline
\end{tabular}

TABLE 3: Odds of association for disposition to nursing facilities in Parkinson's disease inpatients.

\section{Discussion}

Our study aimed to discern the differences in demographics and DBS utilization patterns among PD inpatients with SUD and evaluate the impact of the spectrum of SUD on functionality and discharge disposition among hospitalized patients with PD. We found that alcohol use disorder was most prevalent (82.2\%) and opioid use disorder being the least prevalent. We found that blacks, males and advanced age, and SUD (except cannabis use) were significantly associated with higher odds of major loss of functioning in patients with PD. Furthermore, advanced age, blacks, and females were associated with a higher odd for discharge to SNF/INF.

A higher proportion of male patients with PD had comorbid SUD, with a male: female ratio highest for stimulants (9:1), alcohol (4:1), and opioid and barbiturate (2:1). In general, SUD are more common in males than females due to biological and environmental predispositions [8,9]. Previous studies also found that motor dysfunction is correlated with increased use of alcohol, opioid, and stimulants, and is associated with a functional deficit of the nigrostriatal system leading to increased disease severity in patients with PD 
$[10,11]$. The prevalence of SUD varies with gender, and so is the impact on disease severity and disposition of PD patients. Male patients with PD are more likely to have major loss of functioning by $16 \%$ than females, which is supported by existing literature as females have better improvements in mobility and cognition [12-14].

When a higher proportion of barbiturate use disorder was seen in the elderly population (above 80), then other SUD were prevalent in older adults (60-79 years), and two-third of the PD patients with cannabis use disorder were middle age (40-59 years). Furthermore, we found a strong association between older age (above 80 years) and major loss of functioning (increases by six times). These findings are consistent with results from past studies, which show that advanced age remains a significant risk factor for increased severity of PD even after adjusting for disease duration $[12,15]$. PD is a progressive neurodegenerative disease, and as neuronal loss progresses, disease severity is expected to worsen with age. We also found a strong association between advancing age and a higher likelihood of disposition to nursing facilities. Sharma et al. found that the odds for adverse disposition requiring institutionalized care were more likely in elderly PD patients (>65 years) [16].

Black patients with PD were $68 \%$ more likely to have major loss of functioning compared to whites. Prior research has evaluated racial disparities in outcomes for PD patients. Hemmings et al. reported greater severity of parkinsonian signs, symptoms, and disability in blacks with PD compared to whites. They also noted significant racial differences in the management of PD as blacks were less likely to receive antiparkinsonian medications and less likely to receive the newer medications on the market. Other possible factors include differences in accessing healthcare and socioeconomic status [17]. Dahodwala et al. also reported that although blacks were less likely to be diagnosed with PD, they were four times less likely to receive PD treatment than whites [18]. We found that blacks were $49 \%$ more likely to require discharge to SNF/INF than whites. While there is no prior research on racial differences in discharge disposition among PD patients, one possible reason for this is the higher likelihood of major PD severity among blacks observed in our study.

Regarding SUD among PD patients, we observed that alcohol use disorder was the most prevalent (82\%) and was associated with 1.6 times increased odds for major loss of functioning and adverse disposition to SNF/INF by two times. One explanation for this is the likelihood of worsened PD due to alcohol-induced oxidative stress leading to neurotoxic brain damage $[19,20]$. Another potential reason could be the finding of depleted nigrostriatal monoaminergic terminals in the brains of chronic alcoholics with or without loss of neurons from the substantia nigra [21]. While our results do not imply causality, it adds to the existing literature and should prompt further research regarding the relationship of alcohol use disorder and PD and its impact on patient outcomes.

Furthermore, our data analysis shows that except for cannabis use disorder, all other comorbid SUD were associated with significantly higher odds for major loss of functioning among PD patients. The reason for this lower odd of association with cannabis use remains unclear. Cannabis may show promise in PD as a form of medical marijuana. Current evidence is limited to animal studies which show that cannabis possesses neuroprotective properties against the progressive degeneration of nigrostriatal dopaminergic neurons occurring in PD and may attenuate some of the motor symptoms associated with PD. However, further randomized clinical trials and longitudinal studies are required to determine the long-term benefits and consequences of cannabis use in PD patients [22].

We also found significant relationships between SUD and PD severity. Comorbid opioid use disorder was associated with almost four-fold higher odds for major loss of functioning. The neurobiochemical derangements in PD compromise multiple neurochemical substrates, including dopamine, norepinephrine, serotonin, acetylcholine, and glutamate systems, resulting in several motor impairments. Currently, therapy is focused on dopamine depletion, which also brings about additional motor dysfunction, including dyskinesias [23,24]. Delta opioid receptors are densely located in the basal ganglia, and studies, although inconclusive, have shown that activating these receptors leads to motor improvements, especially for dyskinesias resulting from the use of 1-dopa $[25,26]$.

Overall, these findings suggest that comorbid SUD among PD inpatients is associated with more severe illness and prolonged care. This may result in higher medical care costs for PD patients and their caregivers. $\mathrm{PD}$ is a progressive degenerative disorder that is associated with a significant economic burden for both patients and caregivers $[27,28]$. Due to the complex and multifaceted nature of PD, a wide range of health professionals are involved in care. Prior research and treatment guidelines have stressed the benefits of a collaborative care model that involves a team of neurologists, psychiatrists, nurses, speech therapists, physiotherapists, social workers, and occupational therapists $[29,30]$.

Our study has some limitations. First, being a cross-sectional study, our results only imply association and cannot offer any information on causality between PD and SUD. Also, utilization of inpatient administrative data (and not patient-level data) as the unit of analysis limits the generalizability for all patients with PD. Therefore, it is recommended that future research utilize clinical data or longitudinal data to evaluate the relationship between SUD and PD. 


\section{Conclusions}

SUD are prevalent among PD patients and are associated with more severe illness with body loss of functioning and prolonged inpatient care with adverse disposition to nursing facilities. Therefore, healthcare providers should screen for and treat SUD among this at-risk population to lessen the disease burden and potentially reduce costs of care. A multidisciplinary care model including collaborative psychiatric and addiction management is required to manage SUD among PD patients to lessen disease severity, slow down the disease progression and improve the patient's quality of life.

\section{Additional Information \\ Disclosures}

Human subjects: Consent was obtained or waived by all participants in this study. Animal subjects: All authors have confirmed that this study did not involve animal subjects or tissue. Conflicts of interest: In compliance with the ICMJE uniform disclosure form, all authors declare the following: Payment/services info: All authors have declared that no financial support was received from any organization for the submitted work. Financial relationships: All authors have declared that they have no financial relationships at present or within the previous three years with any organizations that might have an interest in the submitted work. Other relationships: All authors have declared that there are no other relationships or activities that could appear to have influenced the submitted work.

\section{References}

1. Marras C, Beck JC, Bower JH, et al.: Prevalence of Parkinson's disease across North America. NPJ Parkinsons Dis. 2018, 4:21. 10.1038/s41531-018-0058-0

2. Abbas MM, Xu Z, Tan LCS: Epidemiology of Parkinson's disease-east versus west. Mov Disord Clin Pract. 2018, 5:14-28. 10.1002/mdc3.12568

3. Mursaleen LR, Stamford JA: Drugs of abuse and Parkinson's disease . Prog Neuropsychopharmacol Biol Psychiatry. 2016, 64:209-17. 10.1016/j.pnpbp.2015.03.013

4. Tripathi K, Tripathi R: Parkinson's disease and drug abuse. EC Neurology. 2017, 7:117-27.

5. Gramage E, Herradón G: Connecting Parkinson's disease and drug addiction: common players reveal unexpected disease connections and novel therapeutic approaches. Curr Pharm Des. 2011, 17:449-61. $10.2174 / 138161211795164103$

6. Overview of the national (nationwide) inpatient sample . (2021). Accessed: May 15, 2021: https://www.hcup-us.ahrq.gov/nisoverview.jsp.

7. NIS description of data elements. (2021). Accessed: May 15, 2021: https://www.hcupus.ahrq.gov/db/nation/nis/nisdde.jsp.

8. Sex and gender differences in substance use . (2018). Accessed: May 16, 2021: https://www.drugabuse.gov/publications/research-reports/substance-use-in-women/sex-genderdifferences-in-substance-use.

9. Men more likely than women to face substance use disorders and mental illness . (2019). Accessed: May 5, 2021: https://www.pewtrusts.org/en/research-and-analysis/articles/2019/06/03/men-more-likely-thanwomen-to-face-substance-u....

10. Bettiol SS, Rose TC, Hughes CJ, Smith LA: Alcohol consumption and Parkinson's disease risk: a review of recent findings. J Parkinsons Dis. 2015, 5:425-42. 10.3233/JPD-150533

11. Callaghan RC, Cunningham JK, Sykes J, Kish SJ: Increased risk of Parkinson's disease in individuals hospitalized with conditions related to the use of methamphetamine or other amphetamine-type drugs. Drug Alcohol Depend. 2012, 120:35-40. 10.1016/j.drugalcdep.2011.06.013

12. Szewczyk-Krolikowski K, Tomlinson P, Nithi K, Wade-Martins R, Talbot K, Ben-Shlomo Y, Hu MT: The influence of age and gender on motor and non-motor features of early Parkinson's disease: initial findings from the Oxford Parkinson Disease Center (OPDC) discovery cohort. Parkinsonism Relat Disord. 2014, 20:99-105. 10.1016/j.parkreldis.2013.09.025

13. Lubomski M, Louise Rushworth R, Lee W, Bertram KL, Williams DR: Sex differences in Parkinson's disease. J Clin Neurosci. 2014, 21:1503-6. 10.1016/j.jocn.2013.12.016

14. Cholerton B, Johnson CO, Fish B, et al.: Sex differences in progression to mild cognitive impairment and dementia in Parkinson's disease. Parkinsonism Relat Disord. 2018, 50:29-36. 10.1016/j.parkreldis.2018.02.007

15. Reeve A, Simcox E, Turnbull D: Ageing and Parkinson's disease: why is advancing age the biggest risk factor?. Ageing Res Rev. 2014, 14:19-30. 10.1016/j.arr.2014.01.004

16. Sharma M, Ambekar S, Guthikonda B, Wilden J, Nanda A: Regional trends and the impact of various patient and hospital factors on outcomes and costs of hospitalization between academic and nonacademic centers after deep brain stimulation surgery for Parkinson's disease: a United States Nationwide Inpatient Sample analysis from 2006 to 2010. Neurosurg Focus. 2013, 35:E2. 10.3171/2013.8.FOCUS13295

17. Hemming JP, Gruber-Baldini AL, Anderson KE, Fishman PS, Reich SG, Weiner WJ, Shulman LM: Racial and socioeconomic disparities in parkinsonism. Arch Neurol. 2011, 68:498-503. 10.1001/archneurol.2010.326

18. Dahodwala N, Siderowf A, Xie M, Noll E, Stern M, Mandell DS: Racial differences in the diagnosis of Parkinson's disease. Mov Disord. 2009, 24:1200-5. 10.1002/mds.22557

19. Collins MA: Alkaloids, alcohol and parkinson's disease . Parkinsonism Relat Disord. 2002, 8:417-22. 10.1016/s1353-8020(02)00024-X

20. Zahr NM, Kaufman KL, Harper CG: Clinical and pathological features of alcohol-related brain damage . Nat Rev Neurol. 2011, 7:284-94. 10.1038/nrneurol.2011.42

21. Gilman S, Koeppe RA, Adams KM, et al.: Decreased striatal monoaminergic terminals in severe chronic 


\section{Cureus}

alcoholism demonstrated with $(+)[11 \mathrm{C}]$ dihydrotetrabenazine and positron emission tomography. Ann Neurol. 1998, 44:326-33. 10.1002/ana.410440307

22. Patel RS, Kamil S, Shah MR, Bhimanadham NN, Imran S: Pros and cons of marijuana in treatment of Parkinson's disease. Cureus. 2019, 11:e4813. 10.7759/cureus.4813

23. Olanow CW, Obeso JA: Preventing levodopa-induced dyskinesias. Ann Neurol. 2000, 47:S167-76.

24. Calon F, Grondin R, Morissette M, Goulet M, Blanchet PJ, Di Paolo T, Bédard PJ: Molecular basis of levodopa-induced dyskinesias. Ann Neurol. 2000, 47:S70-8.

25. Huang JZ, Ren Y, Xu Y, et al.: The delta-opioid receptor and Parkinson's disease. CNS Neurosci Ther. 2018, 24:1089-99. 10.1111/cns.13045

26. Piccini P, Weeks RA, Brooks DJ: Alterations in opioid receptor binding in Parkinson's disease patients with levodopa-induced dyskinesias. Ann Neurol. 1997, 42:720-6. 10.1002/ana.410420508

27. Martinez-Martin P, Macaulay D, Jalundhwala YJ, Mu F, Ohashi E, Marshall T, Sail K: The long-term direct and indirect economic burden among Parkinson's disease caregivers in the United States. Mov Disord. 2019, 34:236-45. 10.1002/mds.27579

28. Boland DF, Stacy M: The economic and quality of life burden associated with Parkinson's disease: a focus on symptoms. Am J Manag Care. 2012, 18:S168-75.

29. Giladi N, Manor Y, Hilel A, Gurevich T: Interdisciplinary teamwork for the treatment of people with Parkinson's disease and their families. Curr Neurol Neurosci Rep. 2014, 14:493. 10.1007/s11910-014-0493-1

30. van der Marck MA, Kalf JG, Sturkenboom IH, Nijkrake MJ, Munneke M, Bloem BR: Multidisciplinary care for patients with parkinson's disease. Parkinsonism Relat Disord. 2009, 15:219-23. 10.1016/s13538020(09)70819-3 